\title{
Dissociation of NSC606985 induces atypical ER-stress and cell death in prostate cancer cells
}

\author{
LIPING WANG ${ }^{1,2^{*}}$, PENGCHENG FU $^{1,2^{*}}$, YUAN ZHAO ${ }^{1,3}$, GUO WANG $^{1,3}$, RICHARD YU $^{1}$, \\ $\mathrm{XIN}_{\text {WANG }}{ }^{1}$, ZEHAI TANG $^{1}$, JULIANNE IMPERATO-McGINLEY $^{1}$ and YUAN-SHAN ZHU ${ }^{1-3}$ \\ ${ }^{1}$ Department of Medicine, Weill Cornell Medicine, New York, NY 10065, USA; ${ }^{2}$ Department of Clinical Oncology, \\ The First City Hospital of Chenzhou; ${ }^{3}$ Department of Clinical Pharmacology, \\ Xiangya Hospital, Central South University, Changsha, Hunan, P.R. China
}

Received February 9, 2016; Accepted March 17, 2016

DOI: $10.3892 /$ ijo.2016.3555

\begin{abstract}
Castration-resistant prostate cancer (CRPC) is a major cause of prostate cancer (Pca) death. Chemotherapy is able to improve the survival of CRPC patients. We previously found that NSC606985 (NSC), a highly water-soluble camptothecin analog, induced cell death in Pca cells via interaction with topoisomerase 1 and activation of the mitochondrial apoptotic pathway. To further elucidate the role of NSC, we studied the effect of NSC on ER-stress and its association with NSC-induced cell death in Pca cells. NSC produced a timeand dose-dependent induction of GRP78, CHOP and XBP1s mRNA, and CHOP protein expression in Pca cells including DU145, indicating an activation of ER-stress. However, unlike conventional ER-stress in which GRP78 protein is increased, NSC produced a time- and dose-dependent $\mathrm{U}$-shape change in GRP78 protein in DU145 cells. The NSC-induced decrease in GRP78 protein was blocked by protease inhibitors, $N$-acetyl-
\end{abstract}

Correspondence to: Dr Yuan-Shan Zhu, Department of Medicine/ Endocrinology, Weill Cornell Medicine, 1300 York Avenue, Box 149, Room F233, New York, NY 10065, USA

E-mail: yuz2002@med.cornell.edu

*Contributed equally

Abbreviations: 4-PBA, 4-phenylbutyric acid; ALLN, $N$-acetyl-Lleucyl-L-leucylnorleucinal; ATF6, activating transcription factor 6; CHOP/GAD153, growth arrest- and DNA damage-inducible gene 153; CRPC, castration-resistant prostate cancer; EPO, epoxomicin; ER, endoplasmic reticulum; FBS, fetal bovine serum; GRP78, glucose regulated protein 78; HIF-1, hypoxia-inducible factor 1; IRE1, inositol-requiring enzyme 1; NSC, NSC606985; PERK, pancreatic ER kinase (PKR)-like ER kinase; Top1, topoisomerase 1; TUDCA, tauroursodeoxycholic acid; UPR, unfold protein response; VEGF, vascular endothelial growth factor; XBP1s, splicing X-box-binding protein-1

Key words: autophagy, camptothecin analog, chemical chaperon, chemotherapy, CHOP, endoplasmic reticulum stress, mitochondria, prostate cancer
L-leucyl-L-leucylnorleucinal (ALLN), a lysosomal protease inhibitor, and epoxomicin (EPO), a ubiquitin-protease inhibitor. ALLN, but not EPO, also partially inhibited NSC-induced cell death. However, both 4-PBA and TUDCA, two chemical chaperons that effectively reduced tunicamycin-induced ER-stress, failed to attenuate NSC-induced GRP78, CHOP and XBP1s mRNA expression and cell death. Moreover, knockdown of NSC induction of CHOP expression using a specific siRNA had no effect on NSC-induced cytochrome $c$ release and NSC-induced cell death. These results suggest that NSC produced an atypical ER-stress that is dissociated from NSC-induced activation of the mitochondrial apoptotic pathway and NSC-induced cell death in DU145 prostate cancer cells.

\section{Introduction}

Prostate cancer is the most common diagnosed non-cutaneous cancer and the second leading cause of cancer-related death in American men (1). Similar to many other cancers, once prostate cancer has metastasized, there is no curative therapy. Currently, androgen ablation is the major therapy for advanced prostate cancer. Most patients benefit initially from androgen ablation therapy (2), but the tumor cells eventually develop resistance to androgen ablation, becoming castration-resistant prostate cancer (CRPC), with an invariably fatal outcome (3-6). Currently, the discovery of a viable therapy for CRPC is a high priority in the battle against prostate cancer. Two multicenter clinical trials $(7,8)$ have revealed that combinatorial chemotherapy improved median survival in advanced prostate cancer patients, leading to the FDA approval of such chemotherapy for CRPC treatment and emphasizing the importance of chemotherapy in CRPC.

Camptothecin, a plant alkaloid, was first isolated from the Chinese tree Camptotheca acuminata by Wall and colleagues (9). The elucidation of the camptothecin antitumor mechanism by targeting intranuclear enzyme topoisomerase 1 has made camptothecin a promising new class of anticancer drugs, leading to the development of numerous water-soluble derivatives with fewer side effects (10-12). NSC606985 (NSC), a highly water-soluble camptothecin analog, has recently been demonstrated to produce both caspase-dependent and caspase- 
independent tumor cell death via differential mechanisms $(13,14)$. In a previous study, we demonstrated the antitumor activity of NSC in prostate cancer cells through the interaction with topoisomerase 1 and an activation of the mitochondrial apoptotic pathway (15). However, the molecular pathway of NSC activation of cell apoptosis in prostate cancer cells remains to be further elucidated.

Endoplasmic reticulum (ER) plays an essential role in cell function and survival. Accumulation of unfolded or misfolded proteins in the lumen of the ER activates the unfold protein response (UPR), resulting in ER-stress (16-18). The complex cellular response of ER-stress is mediated through three ER transmembrane systems: pancreatic ER kinase-like ER kinase (PERK), activating transcription factor 6 (ATF6) and inositolrequiring enzyme 1 (IRE1). With the accumulation of unfolded or misfolded proteins, GRP78, a molecular chaperone, dissociates from the three receptors, leading to an activation of the three systems and triggering UPR. The activation of these three systems leads to the alteration of multiple downstream events including an alteration of X-box-binding protein-1 (XBP1) mRNA splicing and an upregulation of C/EBP homologous protein (CHOP) gene expression (Fig. 1). The UPR is a pro-survival response to reduce accumulation of unfolded or misfolded proteins and restore normal ER function (19). However, if ER-stress is prolonged, or if the adaptive response fails, apoptosis ensues (19-21). Cell death induced by ER-stress predominantly occurs via the intrinsic mitochondrial apoptotic pathway mainly mediated through CHOP that downregulates the anti-apoptotic mitochondrial protein Bcl-2 (19). Release of cytochrome $c$ from the mitochondrial intermembrane space to cytosol is a key event in intrinsic cell death and is associated with a loss of mitochondrial transmembrane potential and an opening of the mitochondrial permeability transition pore (22). Thus, an overwhelming ER-stress is linked to cell apoptosis (Fig. 1). In this study, we investigated the effect of NSC on ER-stress and the association of NSC-induced ER-stress and cell death.

\section{Materials and methods}

Reagents. NSC606985 (NSC, glycine, 4-ethyl-3,4,12,14tetrahydro-3,14-dioxo-1H-pyrano[3',4':6,7] indolizino [1,2-b] quinolin-4-ylester, (S)-, monohydrochloride, $\mathrm{C}_{22} \mathrm{H}_{19} \mathrm{~N}_{3} \mathrm{O}_{5} . \mathrm{ClH}$ ) was kindly provided by the Drug Synthesis and Chemistry Branch, Developmental Therapeutic Program, Division of Cancer Treatment and Diagnosis, National Cancer Institute (Bethesda, MD, USA). 4-phenylbutyric acid (4-PBA), and tunicamycin were purchased from Sigma (St. Louis, MO, USA). Tauroursodeoxycholic acid (TUDCA), epoxomicin (EPO) and ALLN were from Calbiochem (San Diego, CA, USA). Fetal bovine serum (FBS), L-glutamine, penicillin and streptomycin were obtained from Gemini Bio-Products (Calabasas, CA, USA). Reagents for real-time PCR such as dNTP Mix (10 mM), M-MLV Reverse Transcriptase, RNase inhibitor, and M-MLV RT 5X buffer were purchased from Promega (Madison WI, USA). Antibodies against GRP78 and CHOP were obtained from Santa Cruz Biotechnology (Santa Cruz, CA, USA), $\beta$-actin antibody from Sigma, and cytochrome $c$ antibody from Pharmingen (San Diego, CA, USA). Fast-start universal SYBR green master (Rox) and
TriPure reagents were purchased from Roche Diagnostic Inc. (Indianapolis, IN, USA). Lipofectamine 2000 and random primers were obtained from Invitrogen (Carlsbad, CA, USA).

Cell cultures. LNCaP,PC3 and DU145 cells (ATCC, Rockville, MD, USA) were respectively cultured in RPMI-1640 and Dulbecco's modified Eagle's minimal essential medium (DMEM) supplemented with $10 \%$ fetal bovine serum (FBS), $2 \mathrm{mM} \mathrm{L}$-glutamine, $50 \mathrm{U} / \mathrm{ml}$ of penicillin and $50 \mu \mathrm{g} / \mathrm{ml}$ streptomycin as previously described (23). Cells were maintained in a $5 \% \mathrm{CO}_{2}-95 \%$ air humidified atmosphere at $37^{\circ} \mathrm{C}$ and cultured in phenol-red free medium with $5 \%$ stripped FBS (Gemini Bio-Products) $24 \mathrm{~h}$ before experiments as described (23).

Cell proliferation assay. To measure viable cells, Pca DU145 cells were plated in 96-well plates $(5,000$ cells/well) and treated with various drugs as indicated at $24 \mathrm{~h}$ after plating. Viable cells were counted using a CellTiter AQueous One Solution Cell Proliferation Assay kit following the manufacturer's instructions (Promega, WI, USA).

Reverse transcription PCR (RT-PCR) and quantitative $R T-P C R$. To measure gene expression, RT-PCR and real-time PCR were performed as previously described $(15,24)$. Briefly, total cellular RNA was isolated using TriPure reagents, and the level of specified RNAs were quantified using a NanoDrop2000C. Reverse transcription was performed following the protocol from Promega with $1 \mu \mathrm{g}$ of total cellular RNA and real-time PCR was carried out according to the protocol from Roche in a PCR mixture containing $0.2 \mu \mathrm{M}$ of each primer, $6 \mu 1$ Platinum ${ }^{\circledR} \mathrm{SYBR}^{\circledR}$ Green qPCR SuperMix-UDG dNTPs and $5 \mu \mathrm{l}$ of diluted RT solution (1:50). Primers used for real-time PCR quantification are listed in Table I. Real-time PCR conditions were set according to the protocol from Roche and performed in the $\mathrm{Eco}^{\mathrm{TM}}$ real-time PCR system (Illumine). Glyceraldehydes-3-phosphate dehydrogenase (GAPDH) or $\beta$-actin was used as an internal control. Differences between control and treated samples were calculated using the $\Delta \Delta \mathrm{Ct}$ method and presented as fold of control.

Western blotting. Western blotting was carried out following standard methods with minor modifications (15). Briefly, cells were harvested and total cellular proteins were extracted using a RIPA buffer (25 mM Tris- $\mathrm{HCl} \mathrm{pH} 7.6,150 \mathrm{mM} \mathrm{NaCI}$, $1 \%$ NP-40, $1 \%$ sodium deoxycholate, $0.1 \%$ SDS). Protein concentrations were measured with a Bio-Rad protein assay following the manufacturer's instructions (Bio-Rad, Hercules, CA,USA).Equal protein was fractionated on a Mini-PROTEAN TGX $^{\mathrm{TM}}$ Precast gel (Bio-Rad 456-8123) and transferred to PVDF membrane using the Trans-blot Turbo system (Bio-Rad). Blots were blocked with TBST buffer [ $500 \mathrm{mM} \mathrm{NaCl}, 20 \mathrm{mM}$ Tris-HCl (pH 7.4), and 0.1\% Tween 20] containing 5\% non-fat dry milk overnight at $4^{\circ} \mathrm{C}$ and then incubated with specified primary antibody in TBST buffer containing 5\% non-fat dry milk for $3 \mathrm{~h}$ at room temperature. After secondary antibody incubation at room temperature for $1 \mathrm{~h}$, the signal was visualized with a SuperSignal West Pico Chemiluminescent kit (Pierce Biotechnology Inc. Rockford, IL, USA), imaged and 


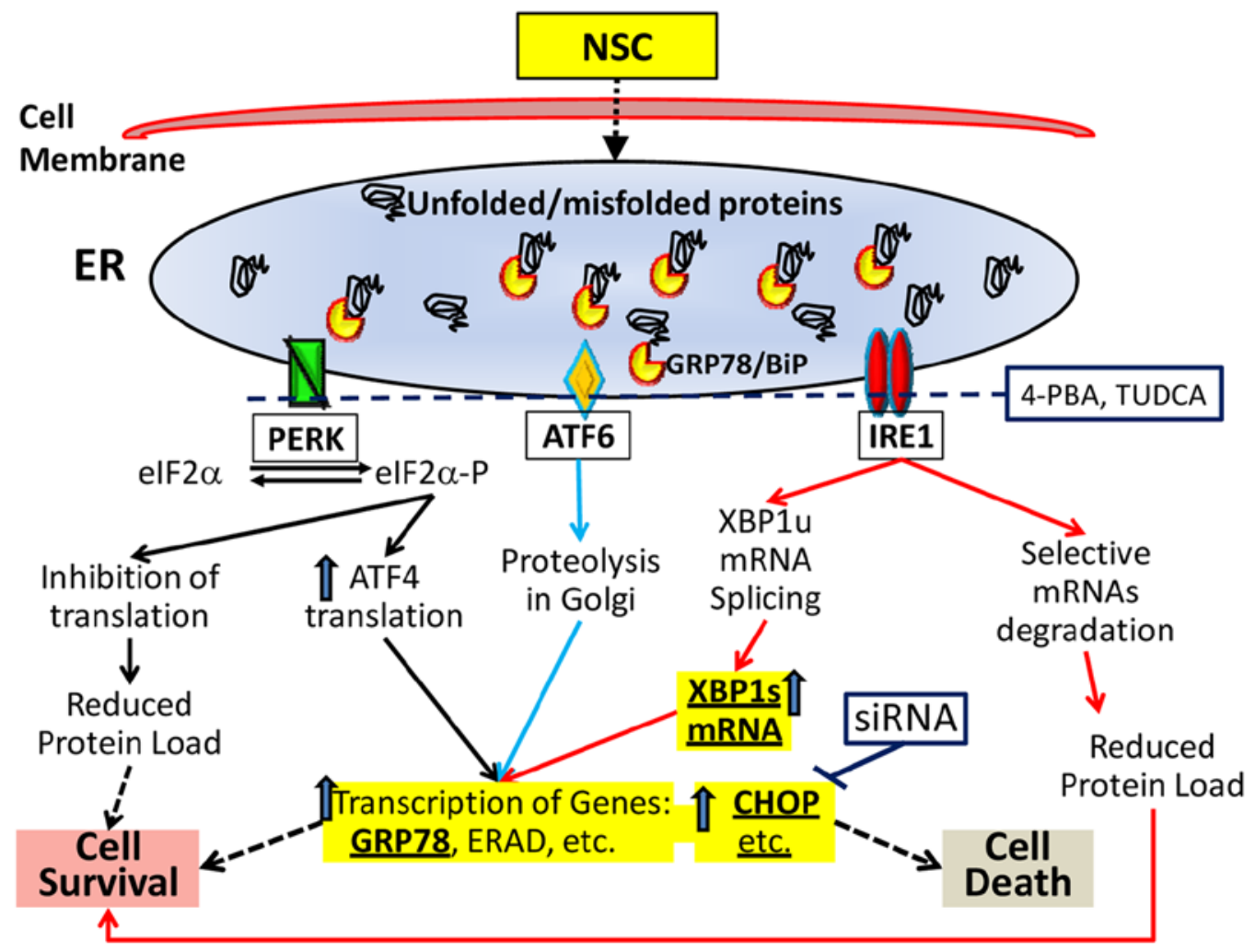

Figure 1. A schematic illustration of NSC-induced UPR and resultant changes in representative ER-stress markers, and other bio-signals related to cell survival and cell death. Major experimental interventions described in this study are also indicated.

Table I. Primers for qRT-PCR.

\begin{tabular}{ll}
\hline Gene & \multicolumn{1}{c}{ Primers } \\
\hline hGRP78 & F: CGAGAACACGGTCTTTGACG \\
& R: ACCACCTTGAACGGCAAGAACT \\
hCHOP & F: 5'-CCTGAGGAGAGAGTGTTCAAG-3' \\
& R: 5'-CTCTTGCAGGTCCTCATACCA-3' \\
hXBP-1 & F: 5'-TTACGAGAGAAAACTCATGGCC-3' \\
& R: 5'-GGGTCCAAGTTGTCCAGAATGC-3' \\
hXBP1s & F: 5'-TGAGAACCAGGAGTTAAGACAGCG-3' \\
& R: 5'-CCTGCACCTGCTGCGGAC-3'
\end{tabular}

hGAPDH F: 5'-GAAGGTGAAGGTCGGAGTC-3'

R: 5'-GAAGATGGTGATGGGATTTC-3'

h $\beta$-actin $\quad$ F: 5'-CTAGAAGCATTTGCGGTGGACGATG-3' R:5'-TCATGAAGTGTGACGTGGACATCCG-3'

F, forward; R, reverse.

quantified with a Bio-Rad ChemoDoc MP system. $\beta$-actin was used as an internal control and detected via incubation of a specific antibody against $\beta$-actin with the same membrane.

Small interference RNA (siRNA) construction and transfection. Based on the human CHOP gene sequences (GenBank accession no. XM_005357988.1), a custom silencer siRNA of
CHOP with a sense sequence of 5'-GAACGGCUCAAGCAG GAAAtt-3' and an antisense sequence of 5'-UUUCCUGCUUG AGCCGUUCat-3', and a negative control silencer siRNA (catalog no. 4390843) were obtained from Invitrogen. For siRNA transfection, DU145 cells were seeded in a 96-well plate, or 12-well plate or 60-mm dishes in phenol red-free DMEM containing 5\% stripped FBS without antibiotics. Twenty-four hours later, the cells were transfected with various concentrations of siRNA (10, 50 and $100 \mathrm{nM})$ using Lipofectamine 2000 according to the manufacturer's instructions (Invitrogen) in OPTI-MEM medium as previously described $(24,25)$. Five hours after transfection, transfection reagents were replaced with DMEM medium and cells were treated with or without various dose NSC for different durations as indicated in each experiment. At the end of the experiments, viable cells were counted with a CellTiter AQueous One Solution Cell Proliferation Assay kit. Knockdown of CHOP expression was verified using quantitative RT-PCR and western blotting.

Analysis of cytochrome c release. Cytosolic cytochrome $c$ was measured as previously described with minor modifications (26). Briefly, cells were pelleted by centrifugation and resuspended in 5X volumes of Buffer A (20 mM HEPES, $10 \mathrm{mM}$ $\mathrm{KCl}, 1.5 \mathrm{mM} \mathrm{MgCl}{ }_{2}, 1 \mathrm{mM}$ EDTA, $1 \mathrm{mM}$ ethylene glycol bis ( $\beta$-aminoethylether)-N,N,N',N'-tetra-acetic acid, 1 mM DTT, $0.1 \mathrm{mM}$ phenylmethylsulfonyl fluoride and complete protease inhibitors) supplemented with $250 \mathrm{mM}$ sucrose. After homogenization with vortexing, the cytosolic fraction was obtained by sequential centrifugations as described (26). Fractionated 


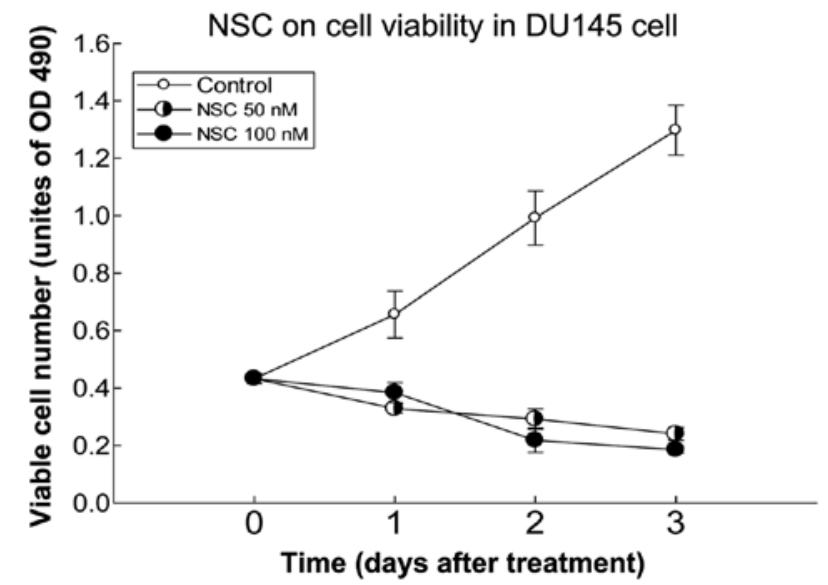

Figure 2. NSC decreased the number of viable cells. DU145 cells were treated with 50 or $100 \mathrm{nM} \mathrm{NSC}$ for indicated times. The number of viable cells was counted as units of absorbance (OD $490 \mathrm{~nm}$ ) and the values are means \pm SEM of twelve individual samples from three independent quadruplicate experiments.

cytosolic proteins were analyzed by western blotting on a Mini-PROTEAN TGX Precast gel as described above.

Statistical analysis. Data are presented as means \pm SEM. One-way analysis of variance (ANOVA) followed by a post hoc Student-Newman-Keuls test and ANOVA on Rank followed by a post hoc Dunn's test were used to determine the difference among multiple groups for parametric and nonparametric data, respectively. A p-value $<0.05$ was considered statistically significant.

\section{Results}

NSC decreases viable DU145 cells. We have previously reported that NSC produced a time- and dose-dependent decrease of cell viability in Pca cells (15). This effect was further verified in this study using DU145 Pca cells (Fig. 2). At a dose of $100 \mathrm{nM}$, NSC produced an $\sim 60 \%$ reduction of viable cells at $72 \mathrm{~h}$ post-treatment compared to pretreatment.

NSC induces ER-stress. To determine whether NSC induces ER-stress in Pca cells, cells were treated with various doses of NSC for different durations ranging from 6 to $72 \mathrm{~h}$, and ER-stress biomarkers including GRP78, CHOP and XBP1s mRNA were determined. In DU145 Pca cells, NSC produced a time-dependent elevation of ER-stress biomarkers, CHOP, XBP1s and GRP78, and their mRNA levels were increased by $>46-$, 11- and 4-fold compared to vehicle control at $36 \mathrm{~h}$ of NSC $(100 \mathrm{nM})$ treatment, respectively (Fig. 3A). Moreover, similar to the CHOP mRNA change, the level of CHOP protein was elevated in a time-dependent manner following
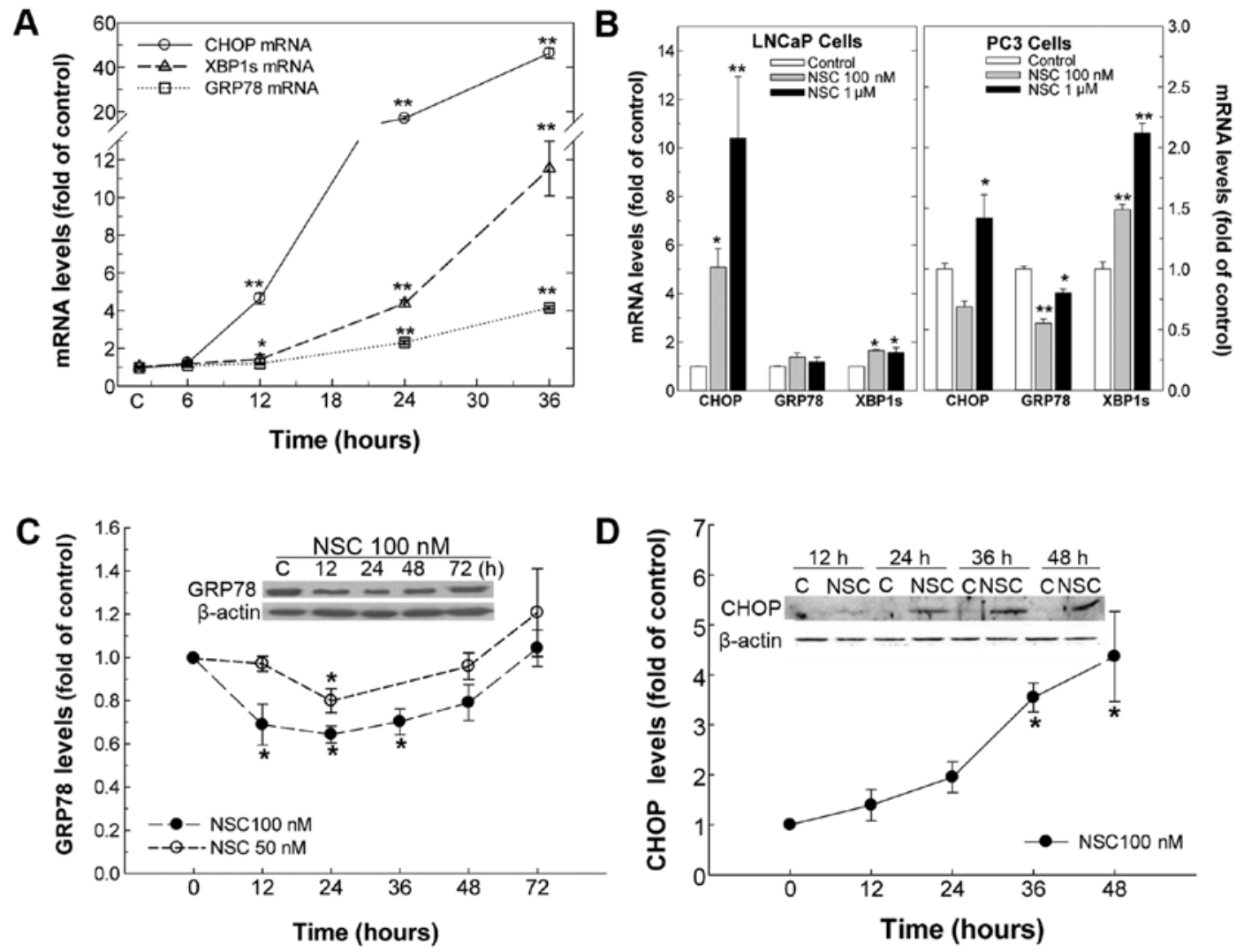

Figure 3. NSC induces changes in ER-stress markers. DU145, LNCaP and PC3 cells were treated with various doses of NSC for various times from 12 to $72 \mathrm{~h}$. Total cellular RNAs and proteins were extracted from the cells and the levels of mRNAs and proteins were determined by real-time RT-PCR and western blotting, respectively. (A) Time-dependent changes in CHOP, XBP1s and GRP78 mRNAs in DU145 cells treated with $100 \mathrm{nM}$ NSC. (B) Dose-dependent changes of CHOP, GRP78 and XBP1s mRNAs in LNCaP and PC cells treated with NSC for $36 \mathrm{~h}$. (C) Representative western blotting and the time- and dosedependent changes of GRP78 protein levels in DU145 cells treated with NSC. (D) Representative western blotting and the quantitative data of NSC-induced time-dependent changes in CHOP protein levels in DU145 cells. The data are means \pm SEM of 3-5 independent experiments. "p $<0.05$ and ${ }^{* *}$ p $<0.01$ compared to the corresponding control group (Student-Newman-Keuls test). 
A

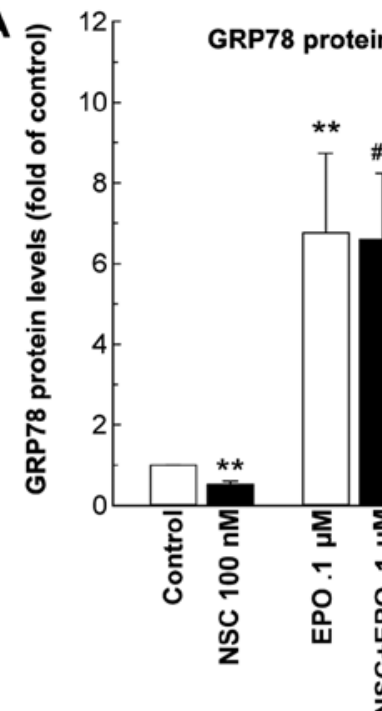

B

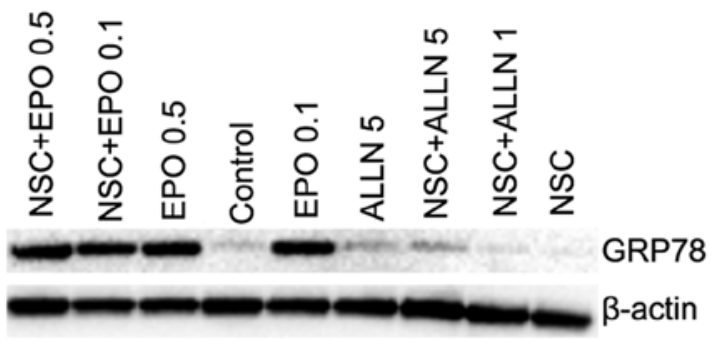

C

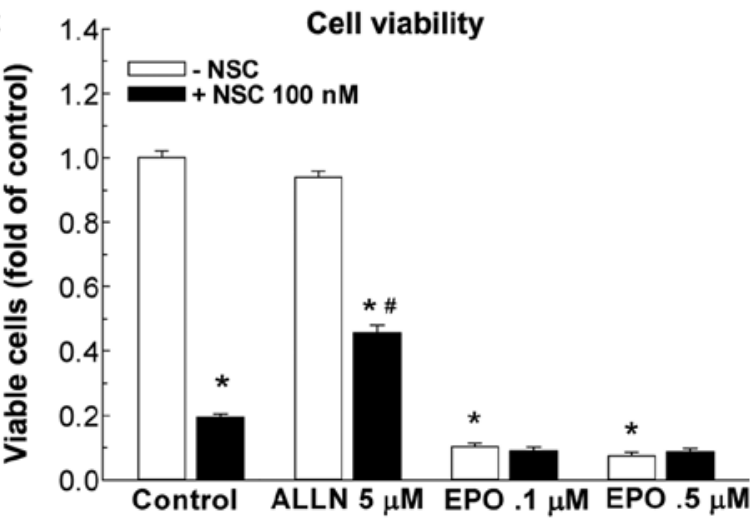

D

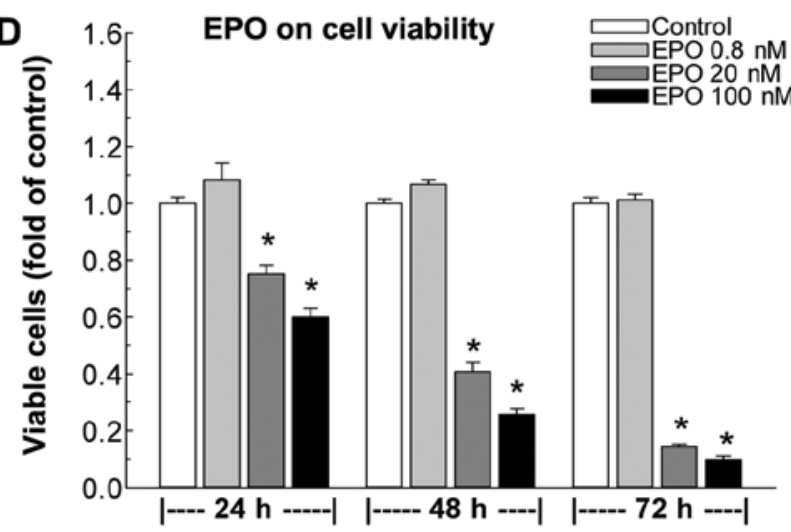

Figure 4. NSC-induced reductions in GRP78 protein (A and B) and viable cell number (C and D) were blocked by protease inhibitors. (A and B) DU145 cells were treated with vehicle control (control), NSC (100 nM), or various doses of EPO and/or ALLN in the indicated combinations for 24 h. Levels of GRP78 protein were determined using western blotting as shown (B) with a representative blot. The values are normalized to the corresponding controls and the data are the mean \pm SEM of 3-6 independent experiments. ${ }^{*} \mathrm{p}<0.05$ and ${ }^{* *} \mathrm{p}<0.01$ compared to control; and ${ }^{\# \#} \mathrm{p}<0.01$ compared to NSC-treated group (StudentNewman-Keuls test). (C and D) DU145 cells were treated with various doses of EPO alone for various times (D), or in combination with NSC (100 nM) for $72 \mathrm{~h}$. Cell proliferation was determined as described in Materials and methods. The data were normalized to the corresponding controls and presented as mean \pm SEM of 4 independent quadruplicate experiments. ${ }^{*} \mathrm{p}<0.05$ compared to control, and ${ }^{\#} \mathrm{p}<0.05$ compared to NSC treatment (Dunn's test)

NSC treatment and a $>4$-fold increase in $\mathrm{CHOP}$ protein was observed at $48 \mathrm{~h}$ of NSC (100 $\mathrm{nM})$ treatment in DU145 cells (Fig. 3D). Surprisingly, unlike the change in GRP78 mRNA, NSC caused a dose- and time-dependent U-shaped change in GRP78 protein. From 12 to $36 \mathrm{~h}$, NSC treatment resulted in a dose- and time-dependent decrease in GRP78 protein, which was gradually recovered by $48-72 \mathrm{~h}$ following NSC treatment (Fig. 3C). Similarly, NSC induced ER-stress in LNCaP and PC3 Pca cells as evident by an increase in ER-stress biomarkers, CHOP and XBP1s mRNA (Fig. 3B).

NSC-induced GRP78 protein reduction and cell death are blocked by protease inhibitors in DU145 cells. To determine if the NSC-induced decrease in GRP78 protein is related to acceleration of protein degradation, two protease inhibitors were employed, EPO that inhibits protein degradation in the ubiquitin-proteasome pathway (27), and ALLN that inhibits protein degradation in the autophagy-lysosomal pathway (28). Treatment of DU145 cells with ALLN at 1 and $5 \mu \mathrm{M}$ doses produced a dose-dependent blockade of NSC-induced GRP78 reduction (Fig. 4A and B) and partially rescued NSC-induced cell death (Fig. 4C). On the contrary, EPO at both 0.1 and $0.5 \mu \mathrm{M}$ concentration inhibited NSC-induced decreases in GRP78 protein levels (Fig. 4A and B) without any effect on NSC-induced cell death (Fig. 4C). Moreover, EPO alone, but not ALLN, markedly increased GRP78 protein levels (Fig. 4A and $\mathrm{B}$ ), while producing a dose- and time-dependent reduction of viable cell numbers (Fig. 4D). At $72 \mathrm{~h}$ post treatment with $100 \mathrm{nM}$ EPO, the viable cell number was reduced by $90 \%$ (Fig. 4D).

NSC-induced ER-stress is not affected by 4-PBA and TUDCA in DU145 cells. TUDCA and 4-PBA have been previously reported to function as chemical chaperons to reduce ER-stress induced by multiple stimuli (29-31). To determine whether NSC-induced ER-stress is inhibited by 4-PBA and TUDCA, DU145 cells were pretreated with different dose 4-PBA or TUDCA for $1 \mathrm{~h}$ and then treated with NSC $(100 \mathrm{nM})$ for $36 \mathrm{~h}$. The doses of 4-PBA and TUDCA were based on previous reports $(32,33)$ and our results in viable cells (Fig. 6). Pretreatment of DU145 cells with 4-PBA (1-3 $\mu \mathrm{M})$ or TUDCA $(2.5-5 \mu \mathrm{M})$ significantly blocked CHOP mRNA 
A

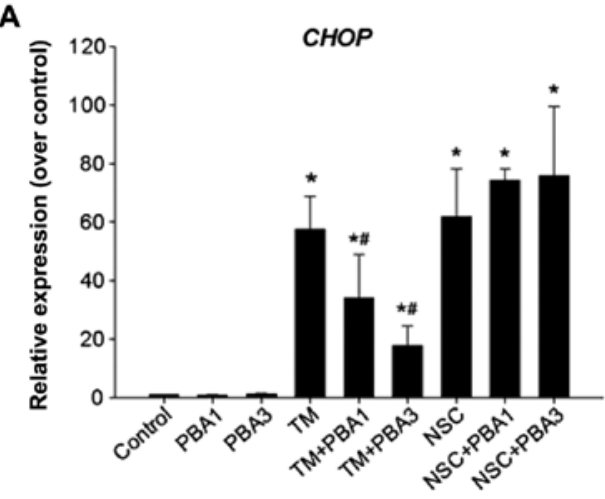

C

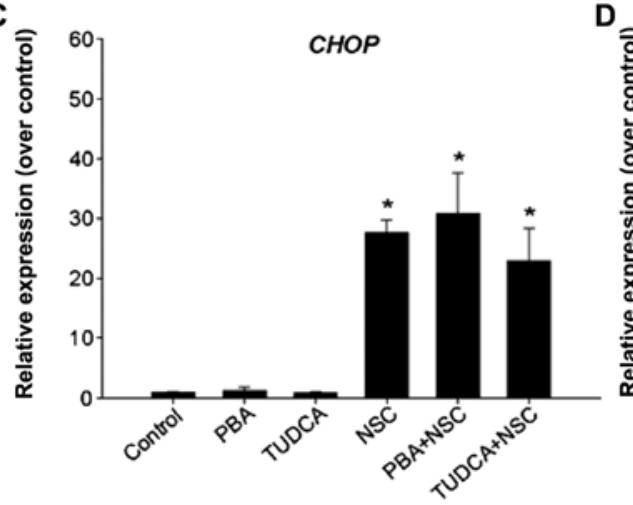

B $\left.\quad{ }^{8}\right] \quad$ GRP78

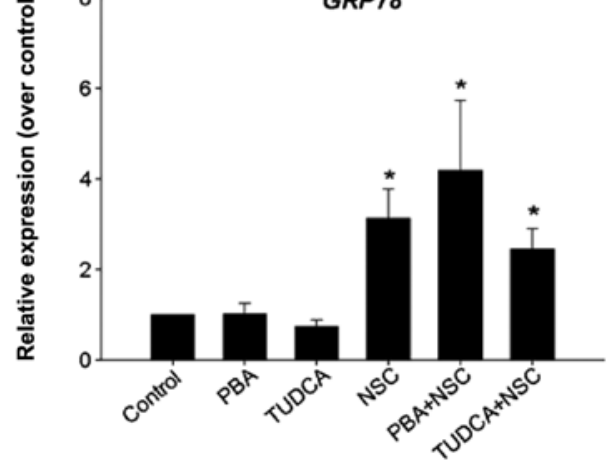

Хर 16

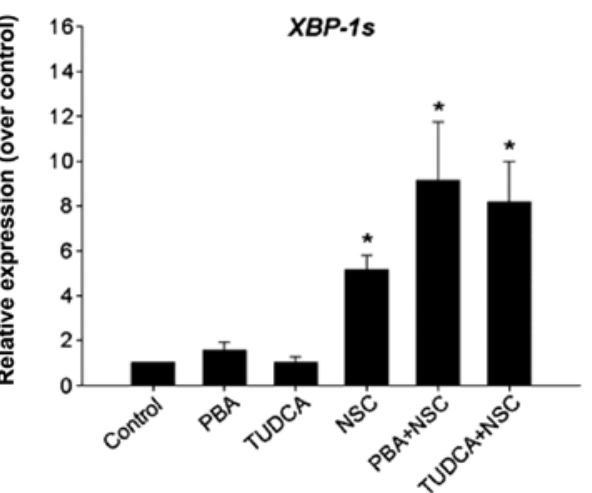

Figure 5 (A-D). Chemical chaperons fail to block NSC-induced ER-stress biomarkers in DU145 cells. DU145 cells were treated with TM (1 $\mu$ g/ml) or NSC $(100 \mathrm{nM})$ for $48 \mathrm{~h}$ in the presence or absence of one hour pretreatment with chemical chaperons, 4 -PBA $(1$ or $3 \mu \mathrm{M})$ or TUDCA ( $2.5 \mu \mathrm{M})$. The levels of GRP78, CHOP and XBP1s mRNAs were determined using real-time RT-PCR as described in Materials and methods. The data were normalized to corresponding controls and presented as mean \pm SEM of 3-5 independent experiments. " $\mathrm{p}<0.05$ compared to the control group, and ${ }^{\#} \mathrm{p}<0.05$ compared to the TM group (Student-Newman-Keuls test).

expression induced by tunicamycin (TM), a positive inducer of ER-stress by disrupting ER $\mathrm{Ca}^{2+}$ homeostasis (34) (Fig. 5A and data not shown). However, neither 4-PBA nor TUDCA inhibited NSC-induced GRP78 (Fig. 5B), CHOP (Fig. 5A and C) and XBP1s mRNA expression (Fig. 5D) in DU145 cells. Thus, NSC-induced ER-stress is differentiated from TM-induced ER-stress because chemical chaperons, 4-PBA and TUDCA failed to block it.

To determine the effect of chemical chaperone 4-PBA and TUDCA on NSC-induced cell death, DU145 cells were pretreated with various concentrations of 4-PBA and TUDCA $1 \mathrm{~h}$ prior to NSC (100 nM) treatment for $72 \mathrm{~h}$. Fig. 6 shows that neither 4-PBA nor TUDCA altered NSC-induced cell death, in agreement with changes in ER-stress biomarkers (Fig. 5). Thus, chemical chaperones, 4-PBA and TUDCA, failed to modify NSC-induced ER-stress and cell death in DU145 cells.

Knockdown of NSC-induced CHOP expression failed to block NSC-induced cytochrome c release and cell death. Because CHOP is a major mediator of ER-stress-induced apoptosis $(35,36)$, we investigated the role of $\mathrm{CHOP}$ in NSC-induced cytochrome $c$ release and cell death. Although transfection of a specific CHOP siRNA markedly reduced basal (Fig. 7B) and NSC-induced CHOP mRNA and protein expression (Fig. 7C-E), it failed to block NSC-induced cytochrome $c$ release from mitochondria to cytosol (Fig. 7F) and NSC-induced cell death (Fig. 7A), indicating that CHOP is not a major mediator of NSC-induced apoptosis and cell death.
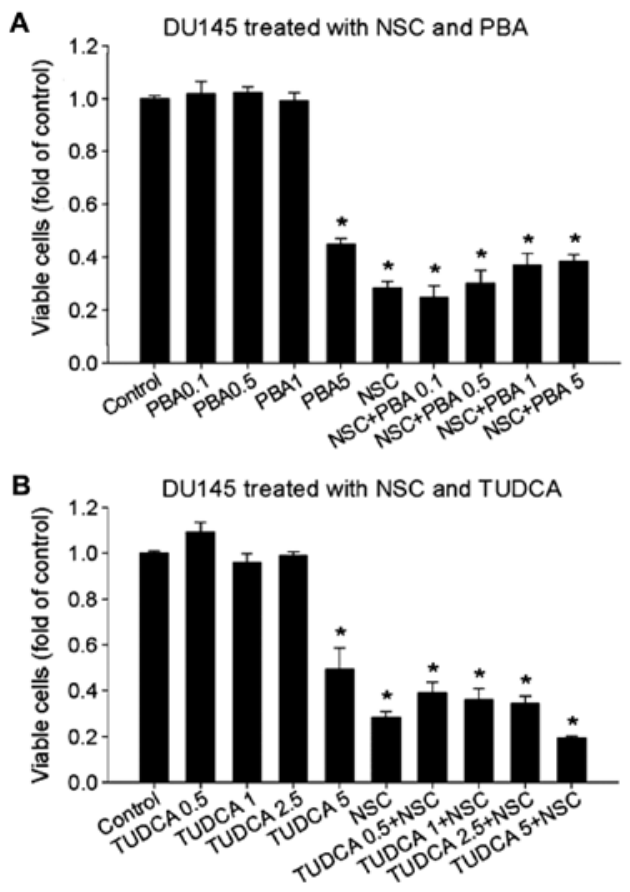

Figure 6. Chemical chaperons fail to alleviate NSC-induced decreases in viable cell numbers. DU145 cells were plated in 96-well plates and treated with vehicle control, $100 \mathrm{nM}$ NSC, or various doses of 4-PBA (A) and TUDCA (B) alone or in combination for $72 \mathrm{~h}$. The viable cell number was determined as described in Materials and methods. The data was normalized to corresponding controls and presented as mean \pm SEM of 3-5 independent quadruplicate experiments. ${ }^{*} \mathrm{p}<0.05$ compared to control group (StudentNewman-Keuls test). 
A
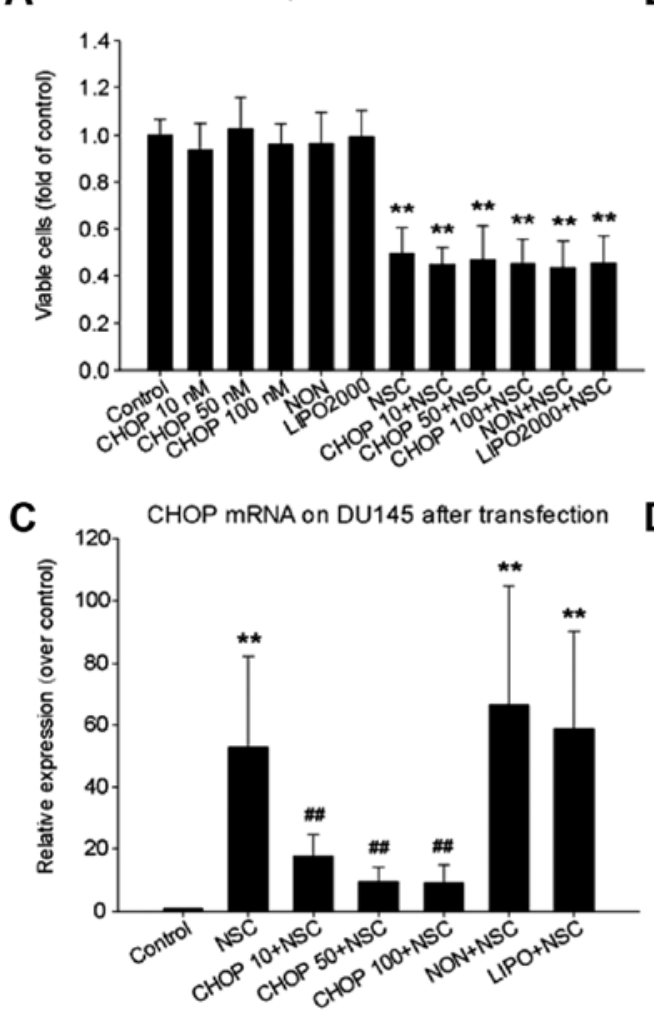

E

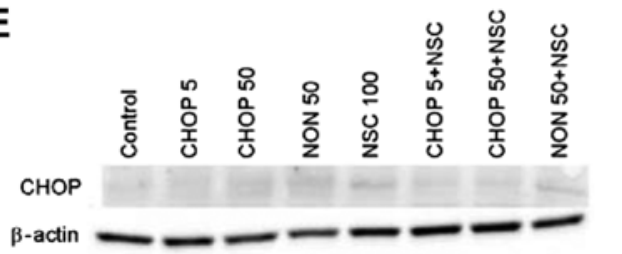

B

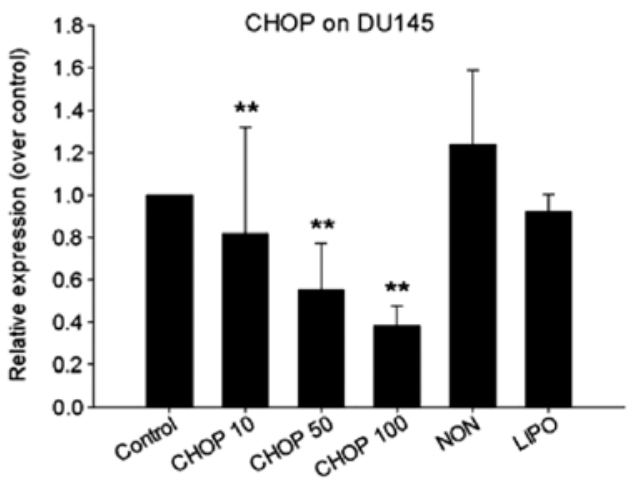

CHOP protein on DU145 after transfection

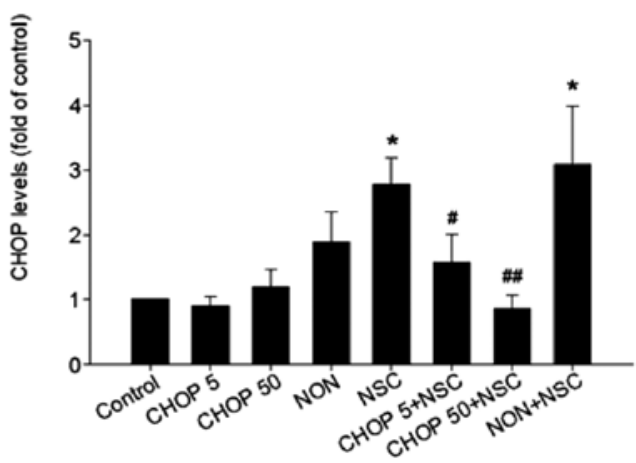

F

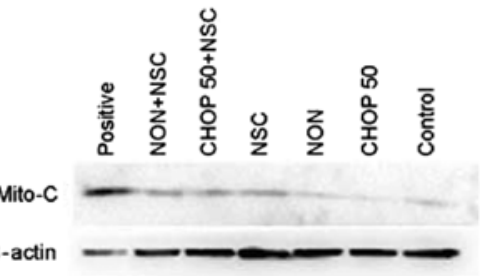

Figure 7. Knockdown of NSC-induced CHOP expression failed to rescue NSC-induced cell death. (A) DU145 cells were transfected with or without various concentrations of a specific CHOP siRNA for $6 \mathrm{~h}$ and then treated with or without NSC $100 \mathrm{nM}$ for $72 \mathrm{~h}$ as indicated. Cell proliferation was determined as described in Materials and methods. (C-E) DU145 cells were pre-transfected with or without various concentrations of a specific CHOP siRNA for $6 \mathrm{~h}$ followed by NSC (100 $\mathrm{nM})$ treatment for $48 \mathrm{~h}$ as indicated. The levels of CHOP mRNA (B and C) and protein (D and E) in the presence or absence of NSC treatment were determined using real-time RT-PCR and western blotting respectively. The data are normalized to corresponding controls and presented as mean \pm SEM of 3-5 independent experiments. (E) Representative CHOP western blotting. (F) Representative western blotting of cytosolic cytochrome $c$ from cells treated with or without a specific CHOP siRNA $(50 \mathrm{nM})$, or NSC $(100 \mathrm{nM})$, alone or in combination. The numbers following CHOP denote the concentration (nM) of the specific CHOP siRNA. NON denotes non-specific siRNA, positive-positive control sample for cytochrome $c$, and LIPO-Lipofactamine 2000 . ${ }^{*}$ p $<0.05$ and ${ }^{* * *} \mathrm{p}<0.01$ compared to corresponding controls; ${ }^{\#} \mathrm{p}<0.05$ and ${ }^{\# \#} \mathrm{p}<0.01$ compared to NSC treatment group (Student-Newman-Keuls test).

\section{Discussion}

In a previous study, we demonstrated that NSC, a watersoluble camptothecin analog, produced a dose- and time-dependent inhibition of cell growth and promotion of cell apoptosis in multiple prostate cancer cell lines (15). In DU145 cells, NSC, through interaction with topoisomerase 1, activated the mitochondrial apoptotic pathway, leading to cytochrome $c$ release from mitochondria into the cytosol and a caspase-dependent cell apoptosis (15). To further elucidate the effect and mechanism of NSC in prostate cancer cells, we studied NSC-induced ER-stress and its association with NSC-induced cell apoptosis in this study. The results indicate that NSC produced atypical ER-stress that was dissociated from NSC-induced cell death.

The ER is an essential cellular organelle responsible for protein synthesis, maturation, folding, transportation, calcium storage, biosynthesis of lipids and sterols (19). ER-stress is the consequence of disequilibrium between the ER folding capacity and the accumulation of unfolded or misfolded proteins in the ER (37). To cope with ER-stress, the cell triggers UPR, initiated via the dissociation of GRP78 from the three ER-stress sensors: PERK, IRE1 $\alpha$ and ATF6 (Fig. 1) $(18,38)$. When PERK is activated, it phosphorylates eukaryotic translation-initiation factor $2 \alpha(\mathrm{eIF} 2 \alpha)$, reducing mRNA translation and biosynthetic protein-folding load in the ER. Once IRE1 $\alpha$ is activated, it functions as an endoribonuclease to remove a 26-nucleotide region from unspliced X-box binding protein 1 (XBP1u) mRNA, generating a spliced XBP1 (XBP1s) mRNA that yields an active transcription factor (XBP1s) to regulate the expression of various subsets of genes involved in protein folding $(18,38)$. On the other hand, the activated IRE1 $\alpha$ causes a degradation of selective mRNAs, resulting in an overall decrease in protein biosynthesis. When ATF6 is activated, it 
can decrease ER protein load via signaling proteolysis in Golgi. These responses collectively result in an upregulation of genes involved in protein folding, maturation and transport such as GRP78, and genes in ER-associated protein degradation (ERAD), leading to a greater chance of cell survival (Fig. 1). However, chronic or overwhelming ER-stress elicits apoptotic signals such as CHOP expression followed by an activation of intrinsic mitochondrial apoptotic pathway $(18,37)$. In this study, we observed that NSC produced a dose- and timedependent elevation of GRP78, XBP1s and CHOP mRNAs (Fig. 3), three ER-stress biomarkers, suggesting that NSC induces ER-stress in Pca cells. However, unlike the classic ER-stress in which GRP78 protein is elevated, NSC produced a time- and dose-dependent U-shaped change in GRP78 protein (Fig. 3C), suggesting that NSC-induced ER-stress in DU145 cells is distinguished from the conventional ER-stress induced by other stimuli.

GRP78, an ER protein chaperone, has greater affinity to unfolded or misfolded proteins and therefore, when these proteins are accumulated in the ER due to various stimuli, GRP78 dissociates from its receptors, triggering the UPR and the activation of three signal pathways (Fig. 1) (18,39). In return, GRP78 as an endpoint marker of the UPR, is regulated by all the three branches of the UPR activated by various stimuli (40-45). GRP78 expression is generally expected to be elevated during ER-stress although the observable increase in protein accumulation usually occurs more slowly than the accumulation of mRNA due in part to the long half-life of GRP78 protein $(37,46)$. In agreement with this concept, the expression of GRP78 mRNA was elevated in a time-dependent manner upon NSC treatment. In contrast, GRP78 protein level significantly decreased between 12 and $36 \mathrm{~h}$, before recovering back to control levels around $72 \mathrm{~h}$ post-NSC treatment, forming a time-dependent U-shaped change (Fig. 3). Although a decrease in GRP78 protein levels is unusual, such ER-stress associated GRP78 reduction has been reported by other investigators in different cells $(47,48)$. It is obvious that the decrease in GRP78 protein is not due to a decrease in GRP78 mRNA since NSC induced a time-dependent increase in GRP78 mRNA levels. We therefore investigated the hypothesis that NSC induces an acceleration of GRP78 protein degradation in DU145 cells. ER associated degradation (ERAD) (Fig. 7), a process which directs unfolded or misfolded proteins for destruction by the cytoplasmic proteasome pathways, is enhanced when cells are subjected to ER-stress $(18,37,49)$. ERAD involves the chaperone of unfolded or misfolded proteins by GRP78 to the cytosol and then degradation of these proteins through either ubiquitin-proteasome or autophagy-lysosome pathway. To determine the role of ubiquitin-proteasome pathway in NSC-induced GRP78 reduction, EPO, a specific ubiquitinproteasome inhibitor (50), was co-administered with NSC in DU145 cells and it prevented to some extent the NSC-induced decrease in GRP78 protein levels (Fig. 4). However, since EPO alone markedly increased GRP78 protein levels, the results do not allow us to conclude that GRP78 degradation via ubiquitin-proteasome pathway alone can explain the NSC-induced reduction in GRP78 protein levels. We then explored a second intracellular pathway of protein degradation, the autophagy-lysosome pathway, a process involving multiple proteases including cysteine protease calpain 1 , cathepsin B and cathepsin L $(28,51,52)$. Using a specific cysteine protease inhibitor, ALLN, we observed that ALLN blocked NSC-induced reduction of GRP78 protein in a dosedependent manner while ALLN alone did not alter GRP78 protein levels (Fig. 4). Taken together, the data suggest that NSC-induced decreases in the GRP78 protein are mediated through protein degradation, mainly the autophagy-lysosome pathway.

It is well documented that ER-stress-triggered UPR activates simultaneously both adaptive and pro-apoptotic pathways (Fig. 1). During chronic or overwhelming ER-stress, the cells enter apoptotic death when the adaptive responses are unable to resolve ER-stress. There are multiple pathways involved in ER-stress-induced cell apoptosis or cell death and the major and best-characterized pathway is through the production of CHOP $(37,49)$. CHOP initiated pro-apoptotic programs involve the downregulation of Bcl2 which leads to outer mitochondrial membrane permeabilization (53), induction of oxidative stress (54), upregulation of death receptor 5 and activation of caspase-8 (55). CHOP expression in most cells is low under non-stressed conditions, and increased in response to ER-stress through IRE1-, PERK-, and ATF6dependent transcriptional induction (Fig. 1) $(18,20,37)$. CHOP has been reported to mediate ER-stress-induced cell apoptosis in prostate cancer cells (56). Overexpression of CHOP induces cell cycle arrest and cell apoptosis in several cell lines, whereas CHOP-deficient cells are protected from ER-stress-induced apoptosis $(20,34,37)$. Taken together, the data suggest that CHOP is a key mediator of ER-stress-related cell apoptosis.

Consistent with the concept of overwhelming ER-stress, CHOP expression at both the mRNA and protein levels was greatly increased following NSC treatment in this study (Fig. 3). To determine whether NSC-induced ER-stress-CHOP activation is involved in NSC-induced cell apoptotic death through an activation of the intrinsic mitochondrial apoptotic pathway (15), two approaches were applied: pretreatment with chemical chaperons and knockdown of CHOP. Chemical chaperones are known to reduce ER-stress by facilitating protein folding and accelerating ER protein trafficking (57). Previous studies have shown that 4-PBA and TUDCA, two chemical chaperons, were able to inhibit ER-stress associated gene expression including CHOP and cell apoptotic death initiated by various stimuli (29,58-61). We therefore pretreated cells with either 4-PBA or TUDA at various doses to test if they inhibit NSC-induced ER-stress and cell death. Unexpectedly, both chemical chaperons failed to inhibit NSC-induced GRP78, XBP1s and CHOP mRNA expression (Fig. 5), and NSC-induced cell apoptotic death (Fig. 6) although they significantly inhibited CHOP mRNA expression stimulated by TM (Fig. 5A and data not shown), a typical ER-stress inducer (34). Furthermore, to define the role of CHOP protein in NSC-induced cell death, a specific CHOP siRNA was used to knockdown CHOP expression. Again, a complete knockdown of NSC-induced CHOP expression did not block NSC-induced cytochrome $c$ release (Fig. 7F) and cell apoptotic death (Fig. 7A). The data collectively suggest that the ER-stress-CHOP pathway is not a major player in modulating NSC-induced cell apoptotic death. The reasons why chemical chaperons, 4-PBA and TUDCA, and CHOP knockdown failed to alleviate NSC-induced changes 
in DU145 cells are currently unknown. It is possible that NSC induces an atypical ER-stress that is not modified by these chemical chaperons. Alternatively, TUDCA and 4-PBA may display other biological actions in addition to their role as inhibitors of the ER-stress response, which may cause complicated unpredictable effects. Finally, the action pathway(s) for NSC induction of this gene expression and cell apoptotic death may completely deviate from the ER stress-CHOP pathway although we cannot exclude the possibility that low levels of $\mathrm{CHOP}$ is sufficient to induce cell apoptosis.

It is interesting to note that blockade of NSC-induced GRP78 protein reduction by ALLN resulted in a partial recovery of NSC-induced cell death (Fig. 4C), suggesting that a decrease in GRP78 protein and/or an activation of the autophagy pathway may involve NSC-induced cell death. Previous studies have indicated that GRP78 was an anti-apoptotic factor (46), and GRP78 overexpression was able to protect against camptothecin-induced cell apoptotic death (62). However, an increase in GRP78 protein alone is not sufficient to protect NSC-induced cell death since EPO blocked NSC-induced reduction of GRP78 protein and markedly elevated GRP78 protein levels, but failed to inhibit NSC-induced cell death (Fig. 4C). Actually, EPO alone produced a marked timeand dose-dependent induction of cell death in DU145 cells (Fig. 4D), consistent with previous reports in other systems (63). Taken together, these results support the hypothesis that a partial blockade of NSC-induced cell death by ALLN involves the autophagy pathway. The relationship between NSC-induced ER-stress, intrinsic mitochondrial apoptosis and autophagy is currently under investigation in this laboratory.

In conclusion, this study in Pca cells demonstrated that NSC produced an atypical ER-stress characterized by an elevation of XBP1s and CHOP expression, an opposite change in GRP78 mRNA and protein levels and a failure of alleviation by chemical chaperons. The NSC-induced decrease in GRP78 protein levels is most likely mediated through protein degradation, mainly the autophagy-lysosome pathway. Furthermore, both chemical chaperons and CHOP knockdown failed to inhibit NSC-induced cell apoptosis while inhibition of cysteine proteases partially restored NSC-induced cell death. The data collectively suggest that even though ER-stress is associated with NSC-induced alterations in DU145 cells, it is dissociated from NSC-induced cell apoptotic death. As a result, elevation of GRP78 mRNA levels may be a more reliable marker of ER-stress than protein levels. This study also provides a clue that NSC may activate cell autophagy, an innovative hypothesis that is under investigation.

\section{Acknowledgements}

This study was supported in part by a Cohen Research Fund from the Division of Endocrinology, Weill Cornell Medicine, and a Youth-Talent Fund (N-2013-09) from the First People's Hospital of Chenzhou City in China.

\section{References}

1. Siegel RL, Miller KD and Jemal A: Cancer statistics, 2015. CA Cancer J Clin 65: 5-29, 2015.

2. Hellerstedt BA and Pienta KJ: The current state of hormonal therapy for prostate cancer. CA Cancer J Clin 52: 154-179, 2002
3. Bubendorf L, Schöpfer A, Wagner U, Sauter G, Moch H, Willi N, Gasser TC and Mihatsch MJ: Metastatic patterns of prostate cancer: An autopsy study of 1,589 patients. Hum Pathol 31: 578-583, 2000.

4. Heidenreich A, Aus G, Bolla M, Joniau S, Matveev VB, Schmid HP and Zattoni F; European Association of Urology: EAU guidelines on prostate cancer. Eur Urol 53: 68-80, 2008.

5. Antonarakis ES and Eisenberger MA: Expanding treatment options for metastatic prostate cancer. N Engl J Med 364: 2055-2058, 2011.

6. Shah RB, Mehra R, Chinnaiyan AM, Shen R, Ghosh D, Zhou M, Macvicar GR, Varambally S, Harwood J, Bismar TA, et al: Androgen-independent prostate cancer is a heterogeneous group of diseases: Lessons from a rapid autopsy program. Cancer Res 64: 9209-9216, 2004.

7. Petrylak DP, Tangen CM, Hussain MH, Lara PN Jr, Jones JA Taplin ME, Burch PA, Berry D, Moinpour C, Kohli M, et al: Docetaxel and estramustine compared with mitoxantrone and prednisone for advanced refractory prostate cancer. N Engl J Med 351: 1513-1520, 2004.

8. Tannock IF, de Wit R, Berry WR, Horti J, Pluzanska A, Chi KN, Oudard S, Théodore C, James ND, Turesson I, et al; TAX 327 Investigators: Docetaxel plus prednisone or mitoxantrone plus prednisone for advanced prostate cancer. N Engl J Med 351: 1502-1512, 2004.

9. Wall ME, Ward EC, Cook CE, Palmer KH, McPhail HT and Sim GA: Plant antitumor agents. I. The isolation and structure of camptothecin, a novel alkaloidal leukemia and tumor inhibitor from Camptotheca acuminata. J Am Chem Soc 88: 3888-3890, 1966.

10. Wall ME: Camptothecin and taxol: Discovery to clinic. Med Res Rev 18: 299-314, 1998.

11. Pizzolato JF and Saltz LB: The camptothecins. Lancet 361: 2235-2242, 2003.

12. Pommier Y: Topoisomerase I inhibitors: Camptothecins and beyond. Nat Rev Cancer 6: 789-802, 2006.

13. Rapisarda A, Uranchimeg B, Scudiero DA, Selby M,Sausville EA, Shoemaker RH and Melillo G: Identification of small molecule inhibitors of hypoxia-inducible factor 1 transcriptional activation pathway. Cancer Res 62: 4316-4324, 2002.

14. Song MG, Gao SM, Du KM, Xu M, Yu Y, Zhou YH, Wang Q, Chen Z, Zhu YS and Chen GQ: Nanomolar concentration of NSC606985, a camptothecin analog, induces leukemic-cell apoptosis through protein kinase Cdelta-dependent mechanisms. Blood 105: 3714-3721, 2005.

15. Tan C, Cai LQ, Wu W, Qiao Y, Imperato-McGinley J, Chen GQ and Zhu YS: NSC606985, a novel camptothecin analog, induces apoptosis and growth arrest in prostate tumor cells. Cancer Chemother Pharmacol 63: 303-312, 2009.

16. Harding HP, Calfon M, Urano F, Novoa I and Ron D: Transcriptional and translational control in the Mammalian unfolded protein response. Annu Rev Cell Dev Biol 18: 575-599, 2002.

17. Rutkowski DT and Kaufman RJ: A trip to the ER: Coping with stress. Trends Cell Biol 14: 20-28, 2004.

18. Binet F and Sapieha P: ER stress and angiogenesis. Cell Metab 22: 560-575, 2015.

19. Schröder M and Kaufman RJ: The mammalian unfolded protein response. Annu Rev Biochem 74: 739-789, 2005.

20. Kim I, Xu W and Reed JC: Cell death and endoplasmic reticulum stress: Disease relevance and therapeutic opportunities. Nat Rev Drug Discov 7: 1013-1030, 2008.

21. Marciniak SJ and Ron D: Endoplasmic reticulum stress signaling in disease. Physiol Rev 86: 1133-1149, 2006.

22. Szegezdi E, Logue SE, Gorman AM and Samali A: Mediators of endoplasmic reticulum stress-induced apoptosis. EMBO Rep 7: 880-885, 2006.

23. Zhu YS, Cai LQ, You X, Cordero JJ, Huang Y and ImperatoMcGinley J: Androgen-induced prostate-specific antigen gene expression is mediated via dihydrotestosterone in LNCaP cells. J Androl 24: 681-687, 2003.

24. Cai J, Hong Y, Weng C, Tan C, Imperato-McGinley J and Zhu YS: Androgen stimulates endothelial cell proliferation via an androgen receptor/VEGF/cyclin A-mediated mechanism. Am J Physiol Heart Circ Physiol 300: H1210-H1221, 2011.

25. Wen J, Zhao Y, Li J, Weng C, Cai J, Yang K, Yuan H, ImperatoMcGinley J and Zhu YS: Suppression of DHT-induced paracrine stimulation of endothelial cell growth by estrogens via prostate cancer cells. Prostate 73: 1069-1081, 2013. 
26. Baydas G, Reiter RJ, Akbulut M, Tuzcu M and Tamer S: Melatonin inhibits neural apoptosis induced by homocysteine in hippocampus of rats via inhibition of cytochrome $c$ translocation and caspase- 3 activation and by regulating pro- and anti-apoptotic protein levels. Neuroscience 135: 879-886, 2005.

27. Kisselev AF and Goldberg AL: Proteasome inhibitors: From research tools to drug candidates. Chem Biol 8: 739-758, 2001.

28. Villalpando Rodriguez GE and Torriglia A: Calpain 1 induce lysosomal permeabilization by cleavage of lysosomal associated membrane protein 2. Biochim Biophys Acta 1833: 2244-2253, 2013.

29. Ozcan U, Yilmaz E, Ozcan L, Furuhashi M, Vaillancourt E, Smith RO, Görgün CZ and Hotamisligil GS: Chemical chaperones reduce ER stress and restore glucose homeostasis in a mouse model of type 2 diabetes. Science 313: 1137-1140, 2006.

30. Benz C, Angermüller S, Töx U, Klöters-Plachky P, Riedel HD, Sauer P, Stremmel W and Stiehl A: Effect of tauroursodeoxycholic acid on bile-acid-induced apoptosis and cytolysis in rat hepatocytes. J Hepatol 28: 99-106, 1998.

31. Kubota K, Niinuma Y, Kaneko M, Okuma Y, Sugai M, Omura T, Uesugi M, Uehara T, Hosoi T and Nomura Y: Suppressive effects of 4-phenylbutyrate on the aggregation of Pael receptors and endoplasmic reticulum stress. J Neurochem 97: 1259-1268, 2006.

32. Luo ZF, Feng B, Mu J, Qi W, Zeng W, Guo YH, Pang Q, Ye ZL, Liu L and Yuan FH: Effects of 4-phenylbutyric acid on the process and development of diabetic nephropathy induced in rats by streptozotocin: Regulation of endoplasmic reticulum stress-oxidative activation. Toxicol Appl Pharmacol 246: 49-57, 2010.

33. Wiley JC, Meabon JS, Frankowski H, Smith EA, Schecterson LC, Bothwell $\mathrm{M}$ and Ladiges WC: Phenylbutyric acid rescues endoplasmic reticulum stress-induced suppression of APP proteolysis and prevents apoptosis in neuronal cells. PLoS One 5: e9135, 2010.

34. Zinszner H, Kuroda M, Wang X, Batchvarova N, Lightfoot RT, Remotti H, Stevens JL and Ron D: CHOP is implicated in programmed cell death in response to impaired function of the endoplasmic reticulum. Genes Dev 12: 982-995, 1998

35. Marciniak SJ, Yun CY, Oyadomari S, Novoa I, Zhang Y, Jungreis R, Nagata K, Harding HP and Ron D: CHOP induces death by promoting protein synthesis and oxidation in the stressed endoplasmic reticulum. Genes Dev 18: 3066-3077, 2004.

36. Watanabe Y, Tsuchiya H, Sakabe T, Matsuoka S, Akechi Y, Fujimoto Y, Yamane K, Ikeda R, Nishio R, Terabayashi K, et al: CD437 induces apoptosis in ovarian adenocarcinoma cells via ER stress signaling. Biochem Biophys Res Commun 366: 840-847, 2008

37. Zhang K and Kaufman RJ: From endoplasmic-reticulum stress to the inflammatory response. Nature 454: 455-462, 2008.

38. Logue SE, Cleary P, Saveljeva S and Samali A: New directions in ER stress-induced cell death. Apoptosis 18: 537-546, 2013.

39. Bertolotti A, Zhang Y, Hendershot LM, Harding HP and Ron D: Dynamic interaction of BiP and ER stress transducers in the unfolded-protein response. Nat Cell Biol 2: 326-332, 2000.

40. Ozcan U, Cao Q, Yilmaz E, Lee AH, Iwakoshi NN, Ozdelen E, Tuncman G, Görgün C, Glimcher LH and Hotamisligil GS: Endoplasmic reticulum stress links obesity, insulin action, and type 2 diabetes. Science 306: 457-461, 2004

41. Ozcan L, Ergin AS, Lu A, Chung J, Sarkar S, Nie D, Myers MG Jr and Ozcan U: Endoplasmic reticulum stress plays a central role in development of leptin resistance. Cell Metab 9: 35-51, 2009.

42. Deldicque L, Cani PD, Philp A, Raymackers JM, Meakin PJ, Ashford ML, Delzenne NM, Francaux M and Baar K: The unfolded protein response is activated in skeletal muscle by high-fat feeding: Potential role in the downregulation of protein synthesis. Am J Physiol Endocrinol Metab 299: E695-E705, 2010.

43. Nivala AM, Reese L, Frye M, Gentile CL and Pagliassotti MJ: Fatty acid-mediated endoplasmic reticulum stress in vivo: Differential response to the infusion of Soybean and Lard Oil in rats. Metabolism 62: 753-760, 2013.

44. Nakamura S, Takizawa H, Shimazawa M, Hashimoto $Y$, Sugitani S, Tsuruma K and Hara H: Mild endoplasmic reticulum stress promotes retinal neovascularization via induction of $\mathrm{BiP} /$ GRP78. PLoS One 8: e60517, 2013.
45. Pierre N, Deldicque L, Barbé C, Naslain D, Cani PD and Francaux M: Toll-like receptor 4 knockout mice are protected against endoplasmic reticulum stress induced by a high-fat diet. PLoS One 8: e65061, 2013.

46. Lee AS: GRP78 induction in cancer: Therapeutic and prognostic implications. Cancer Res 67: 3496-3499, 2007.

47. D'Hertog W, Maris M, Ferreira GB, Verdrengh E, Lage K, Hansen DA, Cardozo AK, Workman CT, Moreau Y, Eizirik DL, et al: Novel insights into the global proteome responses of insulin-producing INS-1E cells to different degrees of endoplasmic reticulum stress. J Proteome Res 9: 5142-5152, 2010.

48. Rosengren V, Johansson H, Lehtiö J, Fransson L, Sjöholm A and Ortsäter H: Thapsigargin down-regulates protein levels of GRP78/BiP in INS-1E cells. J Cell Biochem 113: 1635-1644, 2012.

49. Scheuner D and Kaufman RJ: The unfolded protein response: A pathway that links insulin demand with beta-cell failure and diabetes. Endocr Rev 29: 317-333, 2008.

50. Meng L, Mohan R, Kwok BH, Elofsson M, Sin N and Crews CM: Epoxomicin, a potent and selective proteasome inhibitor, exhibits in vivo antiinflammatory activity. Proc Natl Acad Sci USA 96: 10403-10408, 1999.

51. Bird PI, Trapani JA and Villadangos JA: Endolysosomal proteases and their inhibitors in immunity. Nat Rev Immunol 9: 871-882, 2009.

52. Turk V, Stoka V, Vasiljeva O, Renko M, Sun T, Turk B and Turk D: Cysteine cathepsins: From structure, function and regulation to new frontiers. Biochim Biophys Acta 1824: 68-88, 2012.

53. McCullough KD, Martindale JL, Klotz LO, Aw TY and Holbrook NJ: Gadd153 sensitizes cells to endoplasmic reticulum stress by down-regulating $\mathrm{Bcl} 2$ and perturbing the cellular redox state. Mol Cell Biol 21: 1249-1259, 2001.

54. Tabas I and Ron D: Integrating the mechanisms of apoptosis induced by endoplasmic reticulum stress. Nat Cell Biol 13: 184-190, 2011.

55. Lu M, Lawrence DA, Marsters S, Acosta-Alvear D, Kimmig P, Mendez AS, Paton AW, Paton JC, Walter P and Ashkenazi A: Opposing unfolded-protein-response signals converge on death receptor 5 to control apoptosis. Science 345: 98-101, 2014.

56. Shiraishi T, Yoshida T, Nakata S, Horinaka M, Wakada M, Mizutani Y, Miki T and Sakai T: Tunicamycin enhances tumor necrosis factor-related apoptosis-inducing ligand-induced apoptosis in human prostate cancer cells. Cancer Res 65: 6364-6370, 2005.

57. Engin F and Hotamisligil GS: Restoring endoplasmic reticulum function by chemical chaperones: An emerging therapeutic approach for metabolic diseases. Diabetes Obes Metab 12 (Suppl 2): 108-115, 2010.

58. Ben Mosbah I, Alfany-Fernández I, Martel C, Zaouali MA, Bintanel-Morcillo M, Rimola A, Rodés J, Brenner C, RosellóCatafau J and Peralta C: Endoplasmic reticulum stress inhibition protects steatotic and non-steatotic livers in partial hepatectomy under ischemia-reperfusion. Cell Death Dis 1: e52, 2010.

59. Arrojo E Drigo R, Fonseca TL, Castillo M, Salathe M, Simovic G, Mohácsik P, Gereben B and Bianco AC: Endoplasmic reticulum stress decreases intracellular thyroid hormone activation via an eIF2a-mediated decrease in type 2 deiodinase synthesis. Mol Endocrinol 25: 2065-2075, 2011.

60. Duricka DL, Brown RL and Varnum MD: Defective trafficking of cone photoreceptor CNG channels induces the unfolded protein response and ER-stress-associated cell death. Biochem J 441: 685-696, 2012.

61. Koyama M, Furuhashi M, Ishimura S, Mita T, Fuseya T, Okazaki Y, Yoshida H, Tsuchihashi K and Miura T: Reduction of endoplasmic reticulum stress by 4-phenylbutyric acid prevents the development of hypoxia-induced pulmonary arterial hypertension. Am J Physiol Heart Circ Physiol 306: H1314-H1323, 2014.

62. Reddy RK, Mao C, Baumeister P, Austin RC, Kaufman RJ and Lee AS: Endoplasmic reticulum chaperone protein GRP78 protects cells from apoptosis induced by topoisomerase inhibitors: Role of ATP binding site in suppression of caspase-7 activation. J Biol Chem 278: 20915-20924, 2003.

63. Fu HY, Minamino T, Tsukamoto O, Sawada T, Asai M, Kato H, Asano Y, Fujita M, Takashima S, Hori M, et al: Overexpression of endoplasmic reticulum-resident chaperone attenuates cardiomyocyte death induced by proteasome inhibition. Cardiovasc Res 79: 600-610, 2008. 